\title{
Optomechanical control of molecular motors
}

\author{
David L. Andrews, Luciana C. Dávila Romero, Jamie M. Leeder and Matt M. Coles \\ School of Chemistry, University of East Anglia, Norwich NR4 7TJ, United Kingdom
}

\begin{abstract}
The majority of mechanisms that can be deployed for optical micromanipulation are not especially amenable for extension into the nanoscale. At the molecular level, the rich variety of schemes that have been proposed to achieve mechanical effect using light commonly exploit specific chemical structures; familiar examples are compounds that can fold by cis-trans isomerization, or the mechanically interlocked architectures of rotaxanes. However, such systems are synthetically highly challenging, and few of them can realistically form the basis for a true molecular motor. Developing the basis for a very different strategy based on programmed electronic excitation, this paper explores the possibility of producing controlled mechanical motion through optically induced modifications of intermolecular force fields, not involving the limitations associated with using photochemical change, nor the high intensities required to produce and manipulate optical binding forces between molecules. Calculations reveal that significant, rapidly responsive effects can be achieved in relatively simple systems. By the use of suitable laser pulse sequences, the possibilities include the generation of continuous rotary motion, the ultimate aim of molecular motor design.
\end{abstract}

Keywords: molecular motors, nanomanipulation, optical forces, angular momentum, optomechanical motion

\section{INTRODUCTION}

A macroscopic machine is defined as 'an apparatus using mechanical power and having several parts, each with a definite function and together performing a particular task'. ${ }^{1}$ As a broad definition of concept this can be extended to the micro- and nanoscale regimes, where the basic working principle entails the achievement of linear or rotational motion, depending on specific dynamics and functionality. In a millennial survey of the field, Balzani et al. defined a molecular machine as: '.. an assembly of a distinct number of molecular components that are designed to perform machinelike movements (output) as a result of an appropriate external stimulation (input) ${ }^{2}$. The concept of a machine constructed and operating at the molecular level is not new - nature has built numerous examples into biological systems, and in the life sciences a great deal of current attention focuses on the remarkable properties of such biomolecular motors. A fine example is adenosine triphosphate (ATP) synthase, a cell component with a central role in the storage and delivery of energy. Its well-characterised structure comprises a threefold symmetric rotor straddling the cell membrane. ${ }^{3}$ In the native state the rotor unit turns at $6000 \mathrm{rpm}(100 \mathrm{~Hz})$, delivering or consuming (according to the sense of rotation) three molecules of ATP per rotation; ${ }^{4}$ in the isolated system the mean associated torque is $\sim 20 \mathrm{pN} \mathrm{nm} .{ }^{5}$ The huge interest in such systems is prompted not only by their exquisite design and efficiency; there is clear potential for a range of nanotechnological applications if adequate control can be implemented ex vivo. However, the intrinsic complexity of biomechanical systems and the difficulty of successfully adapting them are very significant obstacles. Moreover, there is an additional tier of complexity in any molecular motor whose action requires the targeted local delivery of fuel chemicals.

Efforts directed towards artificially fabricating and controlling molecular machines or motors have also recently experienced a rapid growth in prominence. As a typical example, self-powered synthetic nanorotors have been prepared from $\mathrm{Au}-\mathrm{Ni}$ nanorods, for which constant-velocity rotations can be induced by the chemical decomposition of hydrogen peroxide at the unattached $\mathrm{Ni}$ end of the rod. ${ }^{6}$ There are also reports of remote-controlled autonomous movement by stripped metallic nanorods. ${ }^{7}$ However, long after such possibilities were first mooted in a notable lecture by Feynman, ${ }^{8}$ there remains a particular interest in optically driven machines - such systems affording numerous advantages over chemically driven equivalents. First, such machines can acquire propulsion energy by a non-contact means, without material fuel; secondly, there is an abundance of sources for the stimulus, including suitably adapted ambient radiation; thirdly, mechanical response to an optical stimulus can be exceptionally fast, and lastly the stimulus is extremely amenable to experimental control. The earliest examples of synthetic machines based on photoisomerization of azobenzene were reported in the early 1980's. ${ }^{9}$ Since then, growing capabilities in molecular synthesis and in

7762 - 1 V. 4 (p.1 of 10) / Color: No / Format: A4 / Date: 2010-07-09 08:17:05 AM

SPIE USE: DB Check, Prod Check, Notes: 
nanofabrication have greatly expanded the feasibility of such 'bottom-up' methodologies. At the genuinely molecular level, the rich variety of schemes that have been proposed to achieve artificial mechanical effect using light commonly exploit specific chemical structures; familiar examples are compounds that fold by cis-trans isomerization, and the abacus-themed architectures of rotaxanes. ${ }^{10,11}$ However, despite being much simpler than biological counterparts, such systems remain synthetically highly challenging, and none of them has been developed sufficiently to form the basis for a realizable motor. Whilst the subject of laser-driven molecular motors has led to some model schemes based on linear motion, ${ }^{12,13}$ at the molecular level there are drawbacks in any unidirectional progressive motion, and schemes geared to rotary action may be considered more attractive for eventual device implementation.

This paper explores a very different strategy, developing the theoretical basis for producing controlled mechanical motion through optically induced modifications of intermolecular force fields, not involving the limitations associated with photochemical change, nor the constraints of deploying materials of biological origin. Calculations and numerical simulations reveal that by programmed electronic excitation, significant, rapidly responsive effects can be achieved in relatively simple systems. By the use of suitable laser pulse sequences, the possibilities include the generation of continuous motion, the ultimate aim of molecular motor design.

\section{MOLECULAR BASIS OF THE THEORY}

Intermolecular interactions are most widely understood in connection with systems in which molecules reside in their electronic ground states ${ }^{14}$ - a reasonable assumption for systems under ambient conditions, when electronically excited state populations are vanishingly small. Even under the conditions that apply in active photochemical experimentation, excited populations are, on the whole, severely limited in spatial and temporal extent. Nonetheless, the nature of attraction between neutral molecules does significantly vary according to electronic state. In particular, the form of the dispersion interaction depends markedly on the electronic states of the molecular or chromophoric participants. The electronic environment produced by the distribution of charge in a chromophore changes upon optical excitation, and the associated modification of electromagnetic interactions with other chromophore units in the vicinity immediately produces modified intermolecular forces. In general, as the system accommodates to the new potential energy field, a degree of local movement can be expected that will close or expand the distance between the species involved. Such a setup has the capacity to produce either linear or rotational motion which is in principle experimentally determinable particularly if one unit is suitably tethered.

The simplest model that describes the interaction of non-polar molecules in their electronic ground state is the LennardJones (LJ) potential, illustrated in Figure 1.

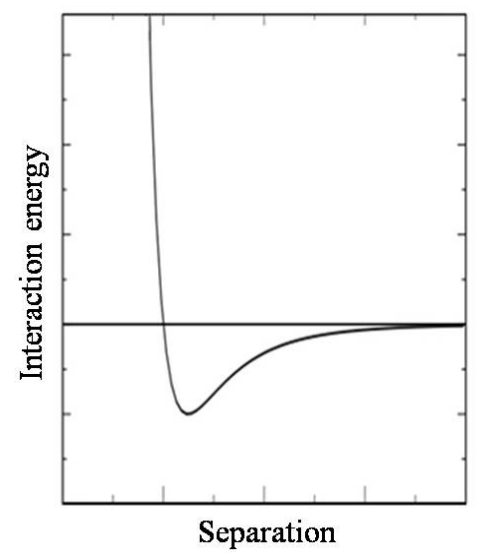

Figure 1. Lennard-Jones potential showing the combined influence of repulsive and attractive contributions, these having $R^{-12}$ and $R^{-6}$ dependences on separation $R$, respectively, between the interacting particles. 
The LJ potential accommodates a repulsive contribution associated with wavefunction overlap at short range, modelled by a $R^{-12}$ dependence on separation $R$, combined with an attractive interaction associated with dispersion interactions. The first descriptions of the latter molecular interactions, beyond the range of significant overlap in electron distribution, resulted in the well-known London formula ${ }^{15}$ or van der Waals interaction, in which the dispersion energy displays an $R^{-6}$ dependence on distance separation. The dispersion forces were originally considered to be the result of second order electrostatic interactions, representing a coupling of fluctuating electric dipoles. Although such a description is physically reasonable and valid at short distances, a theory cast in these terms does not allow for retardation effects necessarily associated with the finite speed of electromagnetic propagation. The most important consequence of including retardation is a replacement of the London dispersion energy distance-dependence by a Casimir-Polder term, derivable using quantum electrodynamics (QED), whose asymptotic form for large intermolecular separations changes to $R^{-7}{ }^{16,17}$ For the purposes to be entertained in the present study, the short-range $R^{-6}$ asymptote of the dispersion interaction suffices.

In the following analysis, our interest will focus upon the varying form of the dispersion interaction between two molecules whose state of electronic excitation varies in time. To this end, we begin with a result for the electrodynamic interaction between a pair of molecular centers denoted generically as $A$ and $B$, with the former in an electronic state $|a\rangle$ and the latter in a state $|b\rangle$. The dispersion potential $\Delta E$, as derived within a QED framework, produces the following expression - see for example reference [18]:

$$
\Delta E=-\frac{1}{24 \pi^{2} \varepsilon_{0}^{2} R^{6}} \sum_{r, s} \frac{\left|\boldsymbol{\mu}^{a r(A)}\right|^{2}\left|\boldsymbol{\mu}^{b s(B)}\right|^{2}}{\left(E_{r a}^{(A)}+E_{s b}^{(B)}\right)},
$$

in which the energy denominator terms are expressed in a concise notation, where for example $E_{r a}^{(A)} \equiv E_{r}^{(A)}-E_{a}^{(A)}$. The parameters $r$ and $s$ represent summed (virtually accessed) electronic levels of $A$ and $B$ respectively. The above expression is generalized in that the physically populated states of $A$ and $B$ are arbitrary; in the case where both $a$ and $b$ represent ground molecular levels, equation (1) describes the London potential. The key physical significance of interpreting the potential when either one or both participating molecules is in an excited state configuration is that the energy denominator can, according to the pair states for which the above expression is evaluated, yield a negative result. ${ }^{19}$ Bearing in mind the sign at the front of equation (1), and the overall dependence on an inverse power of $R$, it transpires that the potential in such cases no longer describes an attractive force, which by contrast is always the case for ground state molecules. In fact, the dispersion coupling can become repulsive, adding to a repulsion due to long-range wavefunction overlap that decays more rapidly with distance; moreover, such an effect persists only as long as the excited state decay lifetime. This is the principle that can be exploited for harnessing the ensuing motion, in a molecular motor.

\section{DESIGN CONSIDERATIONS}

\subsection{Geometric configurations}

In this section we outline the basis for a molecular motor based essentially on one dimensional cyclic movement. In the first case we consider a molecular shuttle in which induced motion is essentially that of a metronome - see Figure 2a). Here a chromophore $B$ oscillates between two chromophores $A$ and $A^{\prime}$ due to their varying attractive/repulsive intermolecular forces. The two drive chromophores are distinct in that the relative positioning of their electronic levels may differ, therefore allowing selective optical excitation of each individually through wavelength-selected laser input. In the second case, which can be considered as a two-dimensional extension of the first, two chromophores of type $B$ perform $360^{\circ}$ rotations and are located at opposite ends of a rigid, non-extendable tether acting as a rotor. Rotor propulsion is achieved by the judicious positioning of four drive chromophores arranged in oppositely positioned equivalent pairs, both for $A$ and $A^{\prime}$, see Figure 2b). In each design the schematic figures are intended only to exhibit the main features of design - the motor 'housing' and 'rotor' for example would necessarily have structural features determined by their fabrication or synthesis. Our primary intention is to identify generic features that the actual 
implementation of any such design must accommodate. The following subsections bring together the various desirable qualities and required properties which facilitate each of the two prototypical designs. The relative arrangements of the chromophore $A$ and $B$ electronic states are considered, together with the relevant temporal parameters and the structural requirements.
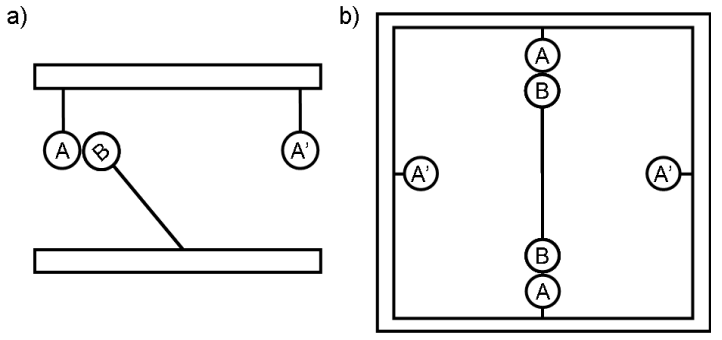

Figure 2. Two schematic configurations for optically driven motion based on programmed electronic excitation of 'drive' chromophores $A$ and rotor chromophores $B$.

\subsection{Relative arrangement of participating electronic levels}

Any comprehensive description of the dispersion force between paired chromophores, as outlined by equation (1), requires consideration of associated intermediate states whose summation accommodates a potentially infinite number of molecular energy levels. For simplicity in the following discussion, it can be assumed that molecular intermediate levels for $A, A^{\prime}$ and $B$ are restricted to only those states through which optical excitation predominantly occur. The choice of accessible molecular levels can then be limited to just the ground and lowest energy excited configuration, $\left|A^{0}\right\rangle$ and $\left|A^{\alpha}\right\rangle$ for $A,\left|A^{\prime 0}\right\rangle$ and $\left|A^{\prime \alpha}\right\rangle$ for $A^{\prime}$ or similarly $\left|B^{0}\right\rangle$ and $\left|B^{\beta}\right\rangle$ for $B$ i.e. a two-level approximation. ${ }^{20}$

It is simplest to elicit the physics by focusing on the $A-B$ pair interaction - the case where $A^{\prime}$ interacts with $B$ follows exactly similar lines, all of the electric dipoles that can feature in the summations of equation (1) will generally comprise either transition dipoles, for example $\boldsymbol{\mu}^{0 \alpha(A)}$ or $\boldsymbol{\mu}^{\alpha 0(A)}$, or static moments of the ground and excited energy levels, such as $\boldsymbol{\mu}^{00(A)}$ and $\boldsymbol{\mu}^{\alpha \alpha(A)}$ - equivalent moments arising for $B$. In the present analysis it is assumed that both rotor and drive chromophores are non-polar, so that the sought motion is not compromised by electrostatic forces. Equation (1) is readily cast to address each of four relevant pair states, specifically $\left(A^{0}-B^{0}\right),\left(A^{\alpha}-B^{0}\right),\left(A^{0}-B^{\beta}\right)$ and $\left(A^{\alpha}-B^{\beta}\right)$, the energy denominators emerging as $\left(E_{\alpha 0}^{(A)}+E_{\beta 0}^{(B)}\right),\left(E_{0 \alpha}^{(A)}+E_{\beta 0}^{(B)}\right),\left(E_{\alpha 0}^{(A)}+E_{0 \beta}^{(B)}\right)$ and $\left(E_{0 \alpha}^{(A)}+E_{0 \beta}^{(B)}\right)$ respectively.

a)

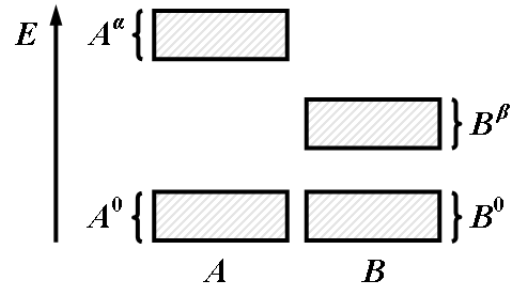

b)

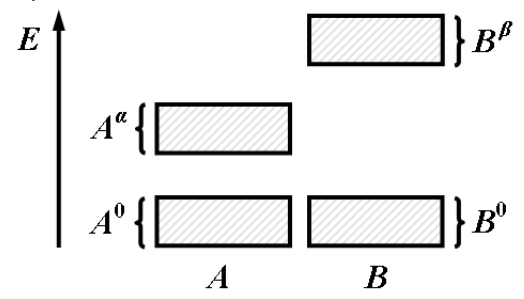

Figure 3. Possible energy level configurations for $A$ and $B$ in a two-level approximation. Both chromophores are limited to their ground and lowest excited state electronic levels, each of which accommodates a continuum of vibrational levels. For a) the LUMO (lowest unoccupied molecular orbital) state of chromophore $A$ is higher in energy than that of $B$, the opposite is portrayed in b). In both cases, there is no overlap between the fluorescence emission and absorption spectra for $A$ and $B$, hence energy transfer processes are precluded.

In determining the attractive or repulsive nature of the dispersion potential for each pair state, it is necessary to consider the relative energy difference between highest occupied and lowest unoccupied molecular orbitals of both $A$ and $B$, i.e. 
the band gap between ground and excited states of each chromophore. Considering first the relative molecular levels portrayed in Figure 3a), where $E_{\alpha 0}^{(A)}>E_{\beta 0}^{(B)}$, it can be shown that an attractive force always persists between the groundstate molecules, since the associated energy denominator remains positive. In contrast, a repulsive force equal in magnitude but opposite in sign to the ground state arrangement is generated if both chromophores are excited. Similarly $\left(A^{0}-B^{\beta}\right)$ and $\left(A^{\alpha}-B^{0}\right)$ pair states respectively represent attractive and repulsive forces of equal, albeit smaller, magnitude than those expected with both chromophores or neither in their ground state.

Physically, for either of the systems portrayed in Figure 2, it can be envisaged that motion of the rotor is affected by an initial excitation of the driver chromophore(s), through optical absorption of radiation that is resonant with $A$, but not $B$. Whilst it might be considered that laser excitation would additionally produce localized movement as a result of radiation pressure, in practice it can be assumed that the usual device of counter-propagating beams can if necessary be deployed as an expedient prevention. Beyond initial excitation, the primary input therefore plays no part in later events, but subsequent optical excitation of $B$ can generate a more significant force, serving to further separate the drive and rotor chromophores. Successful operation of the motor will partially depend on limiting any opportunity in which $A$ resides in a ground state configuration whilst $B$ is excited. Such a pairing would limit the efficiency of the machine, introducing an attractive force in opposition to the desired motion. Addressing this concern, chromophores should thus be chosen such that the decay lifetime of $A^{\alpha}$ significantly exceeds that of $B^{\beta}$. It is interesting to note that in an alternative arrangement depicted in Figure $3 b)$, with $E_{\beta 0}^{(B)}>E_{\alpha 0}^{(A)}$, it is the $\left(A^{0}-B^{\beta}\right)$ pairing instead that generates the initial repulsive force. The general principle is that whichever of $A$ and $B$ has the higher LUMO, should also be required to have the longer decay lifetime. Clearly, similar conclusions for all of these issues can be drawn for the $A^{\prime}-B$ pair interaction.

a)

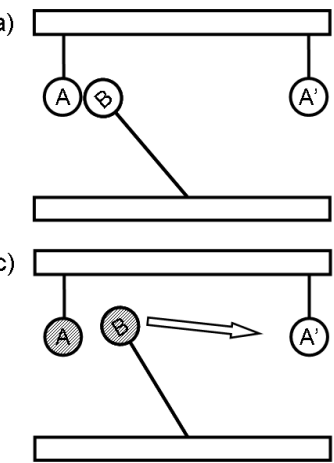

b)

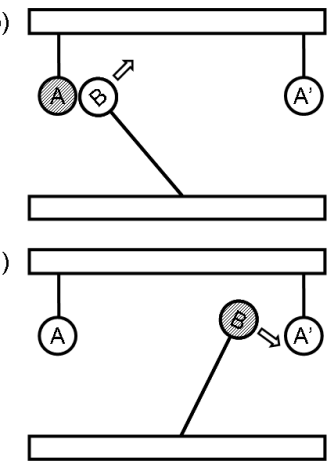

Figure 4. Optically induced motion based upon a molecular shuttle design. From an equilibrium (ground state) configuration a), laser excitation of the drive chromophore modifies the dispersion potential between the drive and rotor chromophores, the latter accordingly experiencing a degree of local movement as portrayed in $b$ ). Assuming the ensuing displacement between $A$ and $B$ remains such that both chromophores can still influence each other, excitation of the rotor chromophore is envisaged to significantly enhance the motion separating the two molecular centres, as observed in c). Finally, as the excited rotor chromophore approaches a second ground state drive chromophore such as in d), an attractive force is anticipated. As the rotor chromphore relaxes to its ground electronic state, an alternative equilibrium configuration is achieved, and an effectively linear displacement motion has been realized.

\subsection{Temporal Parameters}

Based on the presented theory, a number of time-dependent considerations must be addressed to achieve the required motion for both systems outlined in the previous subsection. First, a primary criterion of both configurations is that the decay lifetime of the drive chromophores excited by photon absorption must be sufficiently long, compared to the time in which the two units move significantly apart; Figure 4 illustrates the significance for the simple shuttle motor design. 
a)

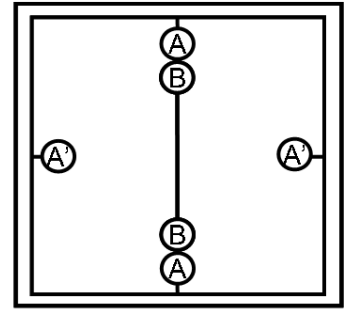

c)

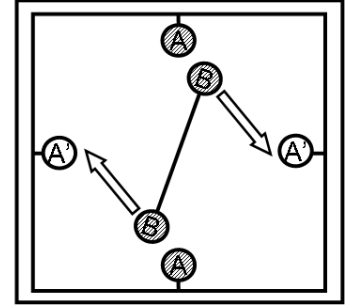

b)

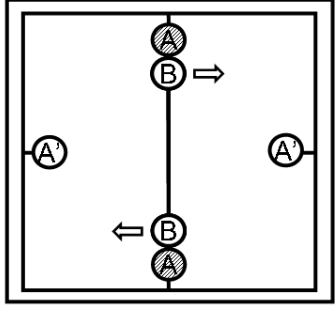

d)

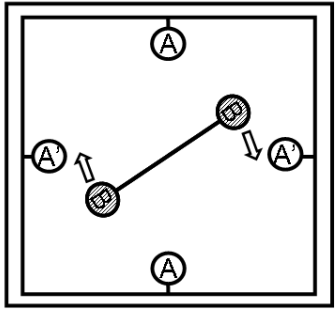

Figure 5. Optically induced motion in a 2-D molecular motor design. From a ground state equilibrium configuration a), the initial displacement of the rotor is induced in a manner fundamentally equivalent to the shuttle design, with the exception that the optical input targets two drive chromophores simultaneously - see b). Similarly, the primary force displacing the rotor from its initial position occurs by the simultaneous excitation of two rotor chromophores whilst in the vicinity of the excited drivers, as in c). Following the attractive force envisaged in d), with ensuing relaxation of the rotor chromophores such that a ground state configuration is achieved, additional $90^{\circ}$ rotations of the rotor are achieved through repetitions of the cycle.

Additional considerations are necessary for the two-dimensional molecular motor system illustrated by Figure 5. Whilst the decay lifetime of the driver must remain sufficiently long compared to the time necessary to significantly separate the drive and rotor chromophores, it must also be shorter than the motor cycle time - which in this case is the time required for a full $180^{\circ}$ rotation of the rotor - in order to preclude the motion that would lead into a reverse-driven status. For an identical reason, the optical pulse duration should itself be short compared to the motor cycle time. Both timescales must, of course, be understood in the statistical sense of a full width at half maximum distribution. Ideally, the pulse repetition interval for the optical excitation (the laser round-trip time in the case of mode-locked pulses) should equal the rotation or cycle time of the motor - although in general, to sustain motor action, the two times need only be synchronized in an integer ratio relationship. Sustained rotation is thus achievable by sequential optical pulses, tailored in wavelength to electronically excite the successively encountered drive chromophores, and having pulse durations and repetition intervals conducive to the sought motion.

\subsection{Structural considerations}

In addition to the previously highlighted geometric, energetic and temporal considerations, it is envisaged that both systems illustrated in Section 3.1 will also equally demand a number of common structural properties, specifically regarding the nature of the rotor. First, as required for any macroscopic machine, the rotor unit needs to have a size that is commensurate with the desired motion, constrained by the associated dependence on mass and moment of inertia. Additionally, subject to stable anchorage, the rotor must clearly exhibit rotational freedom whilst maintaining a degree of structural rigidity. In the absence of such stiffness, any controlled modification to the dispersion force between driver and rotor chromophores may act to distend and bend the moving parts of the system, as opposed to revolving them around a fixed point. These are major considerations when one is dealing with nanoscale systems whose dimensions approach the orders of magnitude associated with the constituent chemical bonds. Clearly, therefore, it is preferable to develop motor systems based on rotors of comparatively small dimensions. As a final consideration, the threedimensional structure of the housing structure will ideally be a highly controllable fabrication process. The external framework on which the motor system is attached, can then be readily adapted such that any achieved motion can be efficiently deployed and utilized for the required mechanical purpose. 


\section{NUMERICAL SIMULATIONS}

Based on the considerations and design criteria detailed in the previous section, it is possible to numerically simulate a model system. We consider here the fundamental component present in both designs, Figure $2 a$ ) and $2 b$ ), consisting of just two chromophores, the fixed $A$ and the potentially mobile but suitably tethered $B$. For simplicity we shall assume that the interaction between the two chromophores, both initially in their ground state, is reasonably well represented by the simple Lennard-Jones model described in Section 3,

$$
V_{L J}(R)=4 \varepsilon\left[\left(\frac{\sigma}{R}\right)^{12}-\left(\frac{\sigma}{R}\right)^{6}\right],
$$

where $R$ is the separation distance. In consequence the two parameters of the potential $V_{L J}(R)$, i.e. $\varepsilon$ and $\sigma$, determine a position of equilibrium that will represent the initial conditions for any motion occurring as a result of changes in electronic states of $A$ and/or $B$. As described in subsection 3.1, to initiate the motor it is assumed that chromophore $A$ is excited; in particular we consider that the band gap between ground and excited states of $A$ is larger than for $B$, as portrayed in figure 3a). Following expression (1) and its subsequent analysis in Section 3.1, it is clear that the attractive $R^{-6}$ force becomes repulsive. Given that the system is initially in a stable state where the $V_{L J}\left(R_{\min }=2^{1 / 6} \sigma\right)=V_{\min }$, it is possible to analyze the dynamics of the rotor molecule, driven by the time-varying change in dispersion potential. If we set the distance between the pivot and the rotor as large compared to the distance the rotor travels, then for calculational simplicity this motion can be approximated as linear. Treating the resulting situation classically - as is widely done in describing the translational motions of molecular systems - we can derive expressions for the dynamics of the rotor. Thus, we assume;

$$
m \frac{d^{2} R}{d t^{2}}=-\frac{d V_{r e p}(R)}{d R}
$$

where $m$ is the mass of the molecule in motion and the repulsive force is given in terms of the potential

$$
V_{r e p}(R)=4 \varepsilon^{\prime}\left(\frac{\sigma}{R}\right)^{6}
$$

It is to be noted that, because of its high inverse power dependence on distance, the $R^{-12}$ component of the repulsion can be considered negligible once the particles move beyond their original equilibrium separation. On solving the differential equation, subject to the given initial conditions, the following expressions for time $t$ and velocity $v$ of the system are obtained, each parametrically expressed in terms of $R$;

$$
\begin{gathered}
t=\frac{1}{6} \sqrt{\frac{m}{\varepsilon^{\prime}}} \sqrt{1-\frac{2 \sigma^{6}}{R^{6}}}\left[3 R-2 \sqrt[6]{2} \sigma F\left(\frac{1}{6}, \frac{1}{2} ; \frac{3}{2} ; 1-\frac{2 \sigma^{6}}{R^{6}}\right)\right] ; \\
v=2 \sqrt{\frac{\varepsilon^{\prime}}{m}} \sqrt{1-\frac{2 \sigma^{6}}{R^{6}}},
\end{gathered}
$$

where the former is cast in terms of the Gaussian hypergeometric function. ${ }^{21}$ Plotting the results by variation of the parameter $R$ gives an illustration of how the rotor velocity varies with time during its initial, accelerative phase - see Figure 6a) and b). Here the physical parameters take values in a range that can be considered typical, rather than bounds. In both expressions it becomes evident that for large separation the velocity $v$ becomes constant and approximately equal 
to $2 \sqrt{\varepsilon^{\prime} / m}$. This asymptotic behavior corresponds to a free motion by the mobile unit $B$, having escaped from the molecular field generated by the drive $A$. In the systems considered earlier, this would constitute a motion onwards, towards the next drive chromophore. Our model takes no account of frictional resistance since at this distance scale, and with this level of representation of the material system, any such effects would be accounted for in terms of other molecular force fields. It is evident that whilst the accelerative phase of motion could be complete within a picosecond, it will generally persist for significantly longer; the subsequent, constant-velocity phase is not subject to any such constraint.

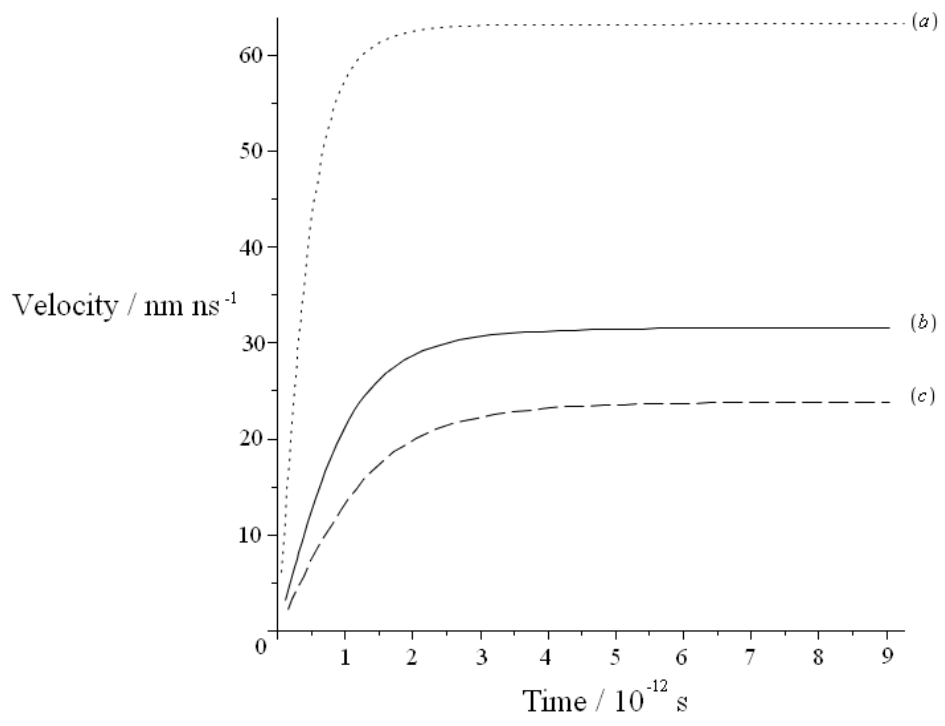

Figure 6a). Plots of escape velocity $v$ vs. time $t$ with varying mass $(a): m=1 \cdot 10^{-24} \mathrm{~kg} ;(b): m=4 \cdot 10^{-24} \mathrm{~kg}$, and (c) $: m=7 \cdot 10^{-24} \mathrm{~kg}$. In each case the $\mathrm{LJ}$ parameters are $\sigma=10^{-10} \mathrm{~m}, \varepsilon=10^{-21} \mathrm{~J}$

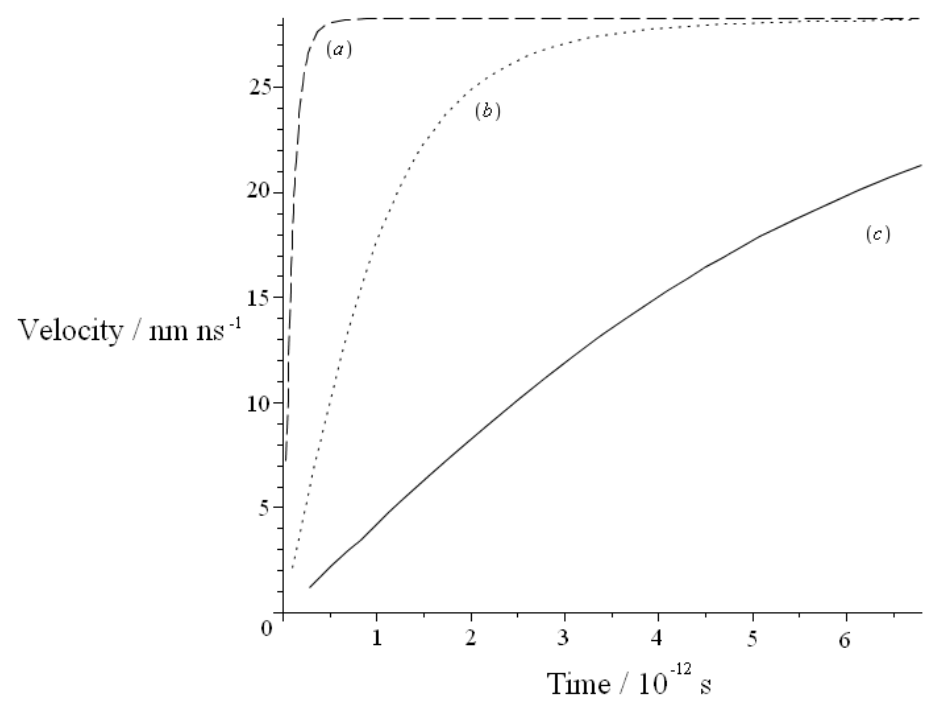

Figure 6b). Plots of escape velocity $v$ vs. time $t$ with varying $\sigma(a): \sigma=1 \cdot 10^{-11} \mathrm{~m}, ;(b): \sigma=5 \cdot 10^{-10} \mathrm{~m}, \quad$ and (c) $: \sigma=1 \cdot 10^{-10} \mathrm{~m}$. In each case $m=5 \cdot 10^{-25} \mathrm{~kg}, \varepsilon=10^{-21} \mathrm{~J}$ 


\section{DISCUSSION}

To the best of our knowledge, this paper represents the first investigation of the possibility to construct molecular motors based on the deployment of direct, optically induced modifications to molecular force fields. The underlying theory is extremely robust, being based on a precise quantum representation of the detailed electronic properties for the key motor parts. In the ensuing development, the first significant approximation is the assumption of a two-level model to simplify the analysis. Such an approximation would be most accurate in application to chromophore species with an unusually low LUMO compared to the positioning of higher unoccupied orbitals: nonetheless it should be emphasised that the assumption is merely an expedient to secure indicative numerical values in the subsequent calculations, and that the same principles would apply even if this approximation were not made. Indeed, it is worth reflecting on what effect the relaxation of such an assumption might have.

It is undoubtedly the case that a more complete sum-over-states calculation for a specific chemical system would not produce the simple change of sign for the $R^{-6} \mathrm{LJ}$ term $\left(R^{-7}\right.$ force), which emerges for the two-level case when one of an interacting pair of chromophores becomes electronically excited. Certainly, it is possible in some systems to secure a complete change from attraction to repulsion. ${ }^{19}$ However, any degree of change in the 'attractive' LJ component, resulting from optical excitation of one component, may prove sufficient. Even if the result of that excitation were only to reduce the size of the attractive force, the $R^{-13}$ repulsive force (from the $R^{-12}$ potential) would impel the mobile $B$ outwards, towards a more distant and shallower point of potential equilibrium. It has to be borne in mind that this repulsion, due to wavefunction overlap (itself never annulled, but potentially enhanced due to a change in electronic state) still operates powerfully in the initial spatial configuration. All that is necessary for the sought motor action to begin, is that the powerfully accelerated $B$ reaches an escape velocity from the molecular field of $A$.

The initial calculations that we have performed, based on this model, indicate forces on the picoNewton scale. More massive rotor structures would produce slower speeds, and rotor velocities in a range that would be tailored to the timescale for onward propulsion of the rotor in one phase of the motor cycle, correlating with achievable pulse repetition times for the optical input. The many variables that feed into the detailed picture suggest the need for multi-variable optimization in the development of principle for application to any specific chemical system. The theoretical evaluation of a measurable displacement remains complex, but it is in principle achievable for any chemical system of interest, through the deployment of a suitable molecular modeling package.

In summary, we have identified realistic prospects for the development of molecular motors based on programmed electronic excitation using pulsed laser light. Without the limitations associated with the use of photochemical change, nor the high intensities required to produce and manipulate optical binding forces between molecules, our calculations have revealed that significant, rapidly responsive effects can be achieved in relatively simple systems. With the use of suitable laser pulse sequences, the possibilities include the generation of continuous rotary motion, the ultimate aim of molecular motor design.

\section{ACKNOWLEDGEMENTS}

This work is funded by the UK Engineering and Physical Sciences Research Council.

\section{REFERENCES}

[1] Soanes, C. and Stevenson, A. eds., [The Oxford Dictionary of English (Revised Edition)], Oxford University Press, Oxford (2005).

[2] Balzani, V., Credi, A., Raymo, F. M. and Stoddart, J. F., “Artificial molecular machines,” Angew. Chem. Int. Ed. 39(19), 3348-3391 (2000).

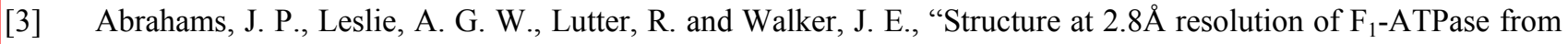
bovine heart mitochondria," Nature 370(6491), 621-628 (1994). 
[4] Cotterill, R. M. J., [Biophysics: An Introduction], Wiley, Chichester (2003).

[5] www.nsti.org/outreach/Biomolecular_Motors/

[6] Fournier-Bidoz, S., Arsenault, A. C., Manners, I. and Ozin, G. A., "Synthetic self-propelled nanorotors," Chem. Commun. (4), 441-443 (2005).

[7] Kline, T. R., Paxton, W. F., Mallouk, T. E. and Sen, A. "Catalytic nanomotors: remote-controlled autonomous movement of striped metallic nanorods," Angew. Chem. Int. Ed. 44(5), 744-746 (2005).

[8] Feynman, R. P., “There's plenty of room at the bottom,” Eng. Sci. 23, 22-36 (1960).

[9] Shinkai, S. and Manabe, O., [Host Guest Complex Chemistry III], Springer Berlin, Heidelberg, 67-104 (1984).

[10] Mahimwalla, Z. S., Ngai, Y. and Barrett, C. J., "Photomechanical effect of azobenzene thin polymer films measured with an AFM cantilever based sensor," Proc. SPIE 7712, 771217 (2010).

[11] Balzani, V., Credi, A. and Venturi, M., "Light powered molecular machines," Chem. Soc. Rev. 38(6), 1542-1550 (2009).

[12] Gehrig, E. and Hess, O., "Spatiotemporal dynamics of optical molecular motors," Phys. Rev. E 68(2), 021914 (2003).

[13] Wang, Z., "Bioinspired laser-operated molecular locomotive," Phys. Rev. E 70(3), 031903 (2004).

[14] Atkins, P. W. and De Paula, J., [Atkins' Physical Chemistry], Oxford University Press, Oxford, Chapter 17 (2010).

[15] London, F., "Zur theorie und systematik der molekularkrafte," Z. Phys. A 63, 245-279 (1930).

[16] Casimir, H. B. O. and Polder, D., "The influence of retardation on the London-van der Waals Forces," Phys. Rev. 73(4), 360-372 (1948).

[17] Salam, A., [Molecular Quantum Electrodynamics: Long-Range Intermolecular Interactions], Wiley, Hoboken, New Jersey (2010).

[18] Andrews, D. L., Bradshaw, D. S., Leeder, J. M. and Rodríguez, J., "Dynamics of the dispersion interaction in an energy transfer system," Phys. Chem. Chem. Phys. 10(34), 5250- 5255 (2008).

[19] Power, E. A. and Thirunamachandran, T., "Dispersion forces between molecules with one or both molecules excited,” Phys. Rev. A 51(5), 3660-3666 (1995).

[20] Meath, W. J., Jagatap, B. N. and Kondo, A. E., "The mechanisms for, and the enhancement of, the simultaneous absorption of two photons by molecules," J. Phys. B 39(15), S605 (2006).

[21] Arfken, G., [Mathematical Methods for Physicists (Third Edition)], Academic Press, London (1985). 